\title{
SIMILARITY BASED IMPULSIVE NOISE REMOVAL IN COLOR IMAGES
}

\author{
$\begin{array}{lll}\text { B. Smolka } & \text { K.N. Plataniotis } & \\ & \text { R. } \text { Lukac }^{3} \quad \text { A.N. Venetsanopoulos }\end{array}$ \\ ${ }^{1}$ Department of Automatic Control, Silesian University of Technology, Poland \\ ${ }^{2}$ The Edward S. Rogers Sr. Department of Electrical and Computer Engineering, \\ University of Toronto, 10 King's College Road, Toronto ON, M5S 3G4, Canada \\ ${ }^{3}$ Slovak Image Processing Center, Jarkova 343, 04925 Dobsina, Slovak Republic \\ ${ }^{4}$ Faculty of Applied Science and Engineering, University of Toronto, \\ 35 St. George Street, Toronto, ON, M5S 3G4, Canada
}

\begin{abstract}
In this paper a novel approach to the problem of impulsive noise removal in color images based on the nonparametric density estimation is presented. The basic idea behind the new image filtering technique is the maximization of the similarities between pixels in a predefined filtering window. The new method is faster than the standard vector median filter and preserves better edges and fine image details. Simulation results show that the proposed method outperforms other standard algorithms of the reduction of impulsive noise in color images.
\end{abstract}

\section{INTRODUCTION}

A number of nonlinear, multichannel filters, which utilize correlation among multivariate vectors using various distance measures, has been proposed [1]-[13]. The most popular nonlinear, multichannel filters are based on the ordering of vectors in a predefined moving window. The output of these filters is defined as the lowest ranked vector according to a specific vector ordering technique.

Let $\mathbf{F}(x)$ represents a multichannel image and let $W$ be a window of finite size $n+1$, (filter length). The noisy image vectors inside the filtering window $W$ are denoted as $\mathbf{F}_{j}, j=0,1, \ldots, n$. If the distance between two vectors $\mathbf{F}_{i}, \mathbf{F}_{j}$ is denoted as $\rho\left(\mathbf{F}_{i}, \mathbf{F}_{j}\right)$ then the scalar quantity $R_{i}=\sum_{j=0}^{n} \rho\left(\mathbf{F}_{i}, \mathbf{F}_{j}\right)$ is the distance associated with the vector $\mathbf{F}_{i}$. The ordering of the $R_{i}$ 's: $R_{(0)} \leq R_{(1)} \leq \ldots \leq$ $R_{(n)}$, implies the same ordering to the corresponding vectors $\mathbf{F}_{i}: \mathbf{F}_{(0)} \leq \mathbf{F}_{(1)} \leq \ldots \leq \mathbf{F}_{(n)}$. Nonlinear ranked type multichannel estimators define the vector $\mathbf{F}_{(0)}$ as the filter output, (Vector Median Filter VMF). However, the concept of input ordering, initially applied to scalar quantities is not easily extended to multichannel data, since there is no universal way to define ordering in vector spaces.

To overcome this problem, distance functions are often utilized to order vectors. The orientation difference between two vectors can also be used as their distance measure. This so-called vector angle criterion is used by the Vector Directional Filters (VDF) to remove vectors with atypical directions [5],[8].

The Basic Vector Directional Filter (BVDF) is a rankedorder, nonlinear filter which parallelizes the VMF operation. However, a distance criterion, different from the $L_{p}$ norm used in VMF, is utilized to rank the input vectors. The output of the BVDF is that vector from the input set, which minimizes the sum of the angles with other vectors. In other words, the BVDF chooses the vector most centrally located without considering the magnitudes of the input vectors.

To improve the efficiency of the directional filters, another method called Directional - Distance Filter (DDF) was proposed [5]. This filter retains the structure of the BVDF but utilizes a new distance criterion to order the vectors inside the processing window.

Another efficient rank-ordered a technique called $\mathrm{Hy}$ brid Directional Filter (HDF) was presented in [9]. This filter operates on the directional and the magnitude of the color vectors independently and then combines them to produce a unique final output.

All standard filters detect and replace well noisy pixels, but their ability of preserving pixels which were not corrupted by the noise process is not sufficient. In this paper we present the construction of a simple, efficient and fast filter which removes noisy pixels, but has the ability of preserving original image pixel values, $[12,13]$.

\section{PROPOSED ALGORITHM}

\subsection{Gray-scale Images}

Let us assume a filtering window $W$ containing $n+1$ image pixels, $\left\{F_{0}, F_{1}, \ldots, F_{n}\right\}$, where $n$ is the number of neighbors of the central pixel $F_{0}$ and let us define the similarity function $\mu:[0 ; \infty) \rightarrow \mathbf{R}$ which is non-ascending in $[0 ; \infty)$, convex in $[0 ; \infty)$ and satisfies $\mu(0)=1$ and $\mu(\infty)=$ 0 . The similarity between two pixels of the same intensity should be 1 , and the similarity between pixels with far dis- 
tant gray scale values should be very close to 0 . The function $\mu\left(F_{i}, F_{j}\right)$ defined as $\mu\left(F_{i}, F_{j}\right)=\mu\left(\left|F_{i}-F_{j}\right|\right)$ satisfies the above conditions.

Let us now define the cumulated sum $M$ of similarities between the pixel $F_{k}$ and all its neighbors. For the central pixel we have $M_{0}$ and for the neighbors of $F_{0}$ we define $M_{k}$ as

$$
M_{0}=\sum_{j=1}^{n} \mu\left(F_{0}, F_{j}\right), \quad M_{k}=\sum_{\substack{j=1 \\ j \neq k}}^{n} \mu\left(F_{k}, F_{j}\right)
$$

which means that for $F_{k}$ which are neighbors of $F_{0}$ we do not take into account the similarity between $F_{k}$ and $F_{0}$ (summation from $j=1$ ) when calculating $M_{k}$, which is the main concept of the new algorithm. The omission of the similarity measure $\mu\left(F_{k}, F_{0}\right)$ privileges the central pixel, as in the calculation of $M_{0}$ we have $\mathrm{n}$ similarities, $\mu\left(F_{0}, F_{k}\right)$, $k=1,2, \ldots, n$ and for $M_{k}, k>0$ we have $n-1$, similarity values, as the central pixel is excluded from the sum $M_{k}$.

In the construction of the new filter, the pixel $F_{0}$ in the window $W$ is replaced by one of its neighbors if $M_{0}<$ $M_{k}, k=1, \ldots, n$. If this is the case, then $F_{0}$ is replaced by that $F_{i}$ for which $i=\arg \max \dot{M}_{i}, i=1, \ldots, n$. In other words $F_{0}$ is detected as being corrupted if $M_{0}<M_{k}, k=$ $1, \ldots, n$ and is replaced by its neighbors $F_{i}$ which maximizes the sum of similarities $M$ between all its neighbors excluding the central pixel, (Figs. refcumukla, 3).

Our basic assumption is that a new pixel must be taken from the window $W$, (introducing pixels which do not occur in the image is prohibited like in the VMF and VDF). For this purpose $\mu$ must be convex, which means that in order to find a maximum of the sum of similarity functions $M$ it is sufficient to calculate the values of $M$ only in points $F_{0}, F_{1}, \ldots, F_{n}$.

\subsection{Color Images}

The presented approach can be applied in a straightforward way to color images. We use the similarity function defined by $\mu\left\{\mathbf{F}_{i}, \mathbf{F}_{j}\right\}=\mu\left(\| \mathbf{F}_{i}-\mathbf{F}_{j}\right) \|$ where $\|\cdot\|$ denotes the specific vector norm. Now, in exactly the same way we maximize the total similarity function $M$ for the vector case.

We have checked several convex similarity functions in order to compare our approach with the standard filters used in color image processing listed in Tab. 1 and we have obtained the best results (Tab. 2, Fig. 2) when applying the following similarity functions, [12]:

$$
\begin{gathered}
\mu_{0}(x)=\exp \left\{-\left(\frac{x}{h}\right)^{2}\right\}, \mu_{1}(x)=\exp \left\{-\frac{x}{h}\right\}, h \in(0 ; \infty), \\
\mu_{2}(x)=\frac{1}{1+x / h}, \mu_{3}(x)=\frac{1}{(1+x)^{h}}, h \in(0 ; \infty),
\end{gathered}
$$

$$
\begin{gathered}
\mu_{4}(x)=1-\frac{2}{\pi} \arctan \left(\frac{x}{h}\right), h \in(0 ; \infty), \\
\mu_{5}(x)=\frac{2}{1+\exp \left\{\frac{x}{h}\right\}}, \mu_{6}(x)=\frac{1}{1+x^{h}}, h \in(0 ; \infty), \\
\mu_{7}(x)=\left\{\begin{array}{cl}
1-x / h & \text { if. } x \leq h, \quad h \in(0 ; \infty) . \\
0 & \text { if } x>h,
\end{array}\right.
\end{gathered}
$$

It is interesting to note, that the best results were achieved for the simplest similarity function $\mu_{7}(x)$, which allows to construct a fast noise reduction algorithm. In the multichannel case, we have

$$
M_{0}=\sum_{j=1}^{n} \mu\left(\mathbf{F}_{0}, \mathbf{F}_{j}\right), \quad M_{k}=\sum_{\substack{j=1 \\ j \neq k}}^{n} \mu\left(\mathbf{F}_{k}, \mathbf{F}_{j}\right)
$$

where $\left.\rho\left\{\mathbf{F}_{i}, \mathbf{F}_{k}\right\}=\| \mathbf{F}_{k}-\mathbf{F}_{l}\right) \|$ and $\|\cdot\|$ is the $L_{2}$ vector norm, as it yields the best results. Applying the linear similarity function $\mu_{7}$ we obtain

$\mu\left(\mathbf{F}_{i}, \mathbf{F}_{k}\right)=\left\{\begin{array}{cl}1-\rho\left(\mathbf{F}_{i}, \mathbf{F}_{k}\right) / h & \text { for } \rho\left(\mathbf{F}_{i}, \mathbf{F}_{k}\right)<h \\ 0 & \text { otherwise }\end{array}\right.$

Then we have from (1)

$$
M_{0}=n-\frac{1}{h} \sum_{j=1}^{n} \rho\left(F_{0}, F_{j}\right)
$$

$$
M_{k}=\sum_{\substack{j=1, j \neq k}}^{n}\left(1-\frac{\rho\left(F_{k}, F_{j}\right)}{h}\right)=n-1-\frac{1}{h} \sum_{j=1}^{n} \rho\left(F_{k}, F_{j}\right)
$$

The difference between $M_{0}-M_{k}$ is

$$
\begin{aligned}
M_{0}-M_{k}= & n-\frac{1}{h} \sum_{j=1}^{n} \rho\left(F_{0}, F_{j}\right)-\left[n-1-\frac{1}{h} \sum_{j=1}^{n} \rho\left(F_{k}, F_{j}\right)\right] \\
& =1-\frac{1}{h} \sum_{j=1}^{n}\left[\rho\left(F_{0}, F_{j}\right)-\rho\left(F_{k}, F_{j}\right)\right]
\end{aligned}
$$

In this way we obtained a condition for retaining the central pixel, as it is considered by the algorithm to be noise-free:

$$
M_{0}-M_{k}>0 \text { if } h>\sum_{j=1}^{n}\left[\rho\left(F_{0}, F_{j}\right)-\rho\left(F_{k}, F_{j}\right)\right]
$$

If this condition is satisfied, then the central pixel is considered as not disturbed by the noise process, otherwise the pixel $\mathbf{F}_{i}$ for which the cumulative similarity value achieves maximum, replaces the central noisy pixel. Thus, the new filter replaces the central pixel only when it is really noisy and preserves the original undistorted image structures.

The parameter $h$ can be set manually by the designer or can be determined adaptively, [12]. 


\begin{tabular}{|c|c|c|}
\hline Notation & Filter & Ref. \\
\hline AMF & Arithmetic Mean Filter & {$[2]$} \\
\hline VMF & Vector Median Filter & {$[7]$} \\
\hline ANNF & Adaptive Nearest Neighbor Filter & {$[11]$} \\
\hline BVDF & Basic Vector Directional Filter & {$[8]$} \\
\hline HDF & Hybrid Directional Filter & {$[9]$} \\
\hline AHDF & Adaptive Hybrid Directional Filter & {$[9]$} \\
\hline DDF & Directional-Distance Filter & {$[5]$} \\
\hline FVDF & Fuzzy Vector Directional Filter & {$[10]$} \\
\hline
\end{tabular}

Table 1. Filters compared.

\begin{tabular}{|c|c|c|c|}
\hline METHOD & NMSE $\left[10^{-4}\right]$ & RMSE & PSNR [dB] \\
\hline NONE & 514.95 & 32.165 & 17.983 \\
\hline AMF & 82.863 & 12.903 & 25.917 \\
\hline VMF & 23.304 & 6.842 & 31.427 \\
\hline ANNF & 31.271 & 7.926 & 30.149 \\
\hline BVDF & 29,074 & 7.643 & 30.466 \\
\hline HDF & 22.845 & 6.775 & 31.513 \\
\hline AHDF & 22,603 & 6,739 & 31.559 \\
\hline DDF & 24.003 & 6.944 & 31,288 \\
\hline FVDF & 26.755 & 7.331 & 30.827 \\
\hline \multicolumn{4}{|c|}{ PROPOSED FILTERING SCHEME } \\
\hline$\mu_{0}(x)$ & 5.056 & 3.163 & 38.137 \\
\hline$\mu_{1}(x)$ & 4.959 & 3.157 & 38.145 \\
\hline$\mu_{2}(x)$ & 5.398 & 3.294 & 37.776 \\
\hline$\mu_{3}(x)$ & 9.574 & 4.387 & 35.288 \\
\hline$\mu_{4}(x)$ & 5.064 & 3.190 & 38.054 \\
\hline$\mu_{5}(x)$ & 4.777 & 3.099 & 38.307 \\
\hline$\mu_{6}(x)$ & 11.024 & 4.707 & 34.675 \\
\hline$\mu_{7}(x)$ & 4.693 & 3.072 & 38.384 \\
\hline
\end{tabular}

Table 2. Comparison of the new algorithm based on different kernel functions with the standard techniques (LENA) contaminated by $4 \%$ of impulsive noise.

\section{CONCLUSIONS}

The new presented algorithm is a modification and improvement of the VMF. The efficiency of the proposed filter has been compared with the standard algorithms of noise reduction, (Tab. 2, Figs. 2 and 4. The comparison shows that the new filter significantly outperforms the standard procedures used in color image processing, when the impulse noise should be eliminated.

\section{REFERENCES}

[1] A.N. Venetsanopoulos, K.N. Plataniotis, Multichannel image processing, Proceedings of the IEEE Workshop on Nonlinear Signal/Image PTocessing, 2-6, (1995)

[2] 1. Pitas, A. N. Venetsanopoulos, 'Nonlinear Digital Filters : Principles and Applications', Kluwer Academic Publishers, Boston, MA. (1990)

[3] K.N. Plataniotis, A.N. Venetsanopoulos, 'Color Image Processing and Applications', Springer Verlag, (June 2000)

[4] I. Pitas, P. Tsakalides, Multivariate ordering in color image processing, IEEE Trans. on Circuits and Systems for Video Technology, 1, 3, 247-256, (1991)

[5] D.G. Karakos, P.E. Trahanias, Combining vector median and vector directional filters: The directional - distance filters, Proceedings of the IEEE Conf. on Image Processing, ICIP-95, 171-174, (October 1995)

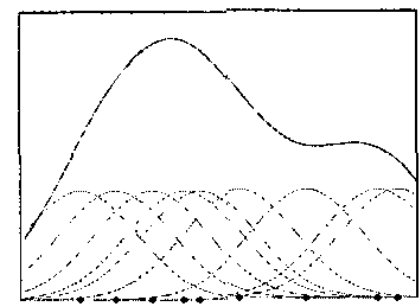

a)

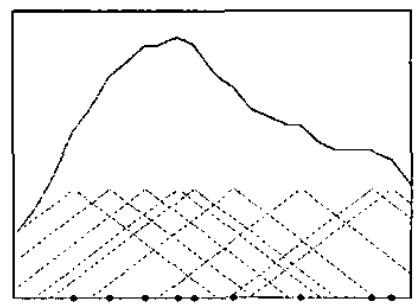

b)
Fig. 1. Cumulative similarity values dependence on the pixel gray scale value for a window containing a set of pixels with intensities $\{15,24,33,41,45,55,72,90,95\}$ using the $\mu_{0}$ function (a) and $\mu_{7}$ function (b).

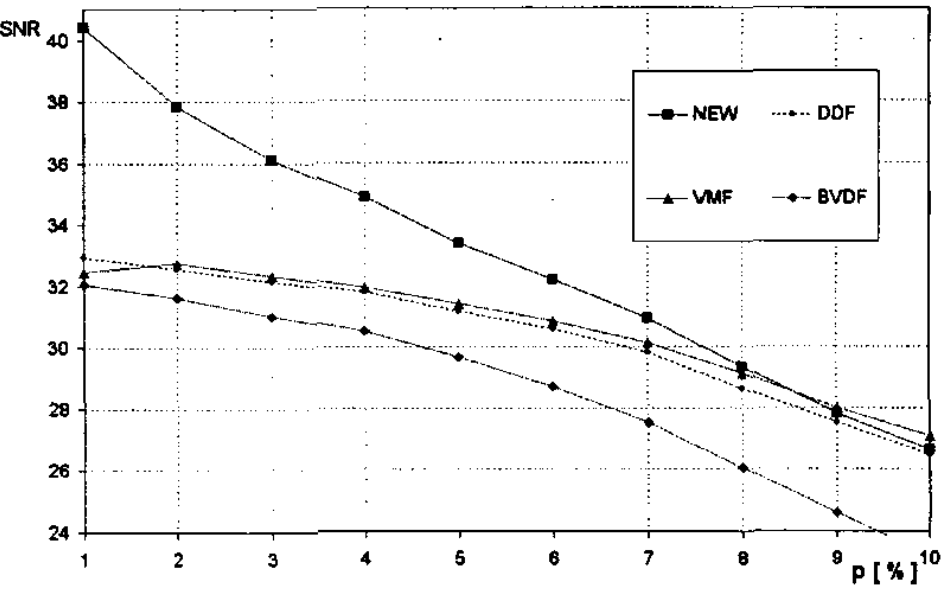

Fig. 2. Comparison of the efficiency of the new filter in terms of PSNR with VMF, BVDF and DDF using the $L E N A$ image corrupted by impulsive noise applied separately on each RGB channel ranging from 1 to $10 \%$.

[6] I. Pitas, A.N. Venetsanopoulos, Order statistics in digital image processing, Proceedings of IEEE, 80, 12, 1893-1923, (1992)

[7] J. Astola, P. Haavisto, Y. Neuovo, Vector median filters, IEEE Proceedings, 78, 678-689, (1990)

[8] P.E. Trahanias, A.N. Venetsanopoulos, Vector directional filters: A new class of multichannel image processing filters, IEEE Trans. on Image Processing, 2, 4, 528-534, (1993)

[9] M. Gabbouj, F.A. Cheickh, Vector Median-Vector Directional hybrid filter for color image restoration, Proceedings of EUSIPCO-96 879-881, (1996)

[10] K.N. Plataniotis, D. Androutsos, A.N. Venetsanopoulos, Colour Image Processing Using Fuzzy Vector Directional Filters, Proceedings of the IEEE Workshop on Nonlinear Signa//mage Processing, Greece, 535-538, (1995)

[11] K.N. Plataniotis, D. Androutsos, V. Sri, A.N. Venetsanopoulos, A nearest neighbour multichannel filter, Electronic Letters, 19101911, (1995)

[12] B. Smolka, A. Chydzinski, K. Wojciechowski, K. Plataniotis, A.N Venetsanopoulos, On the reduction of impulsive noise in multichannel image processing, Optical Engineering, vol. 40, no. 6, pp. 902$908,2001$.

[13] B. Smolka, A. Chydzinski, K. Wojciechowski, K. Plataniotis, A Venetsanopoulos, Self-adaptive algorithm of impulsive noise reduction in color images, Pattern Recohnition, vol. 35, pp. 1771-1784, 2002. 


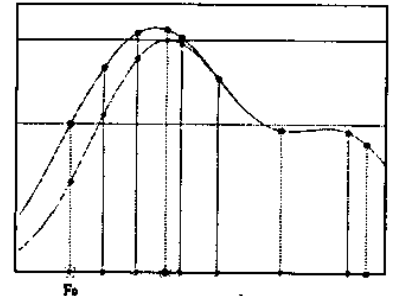

a)

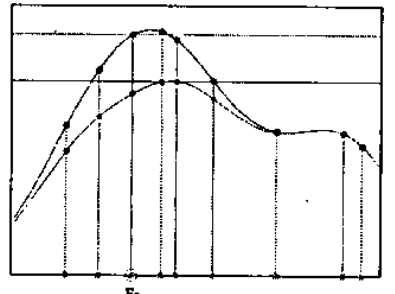

c)

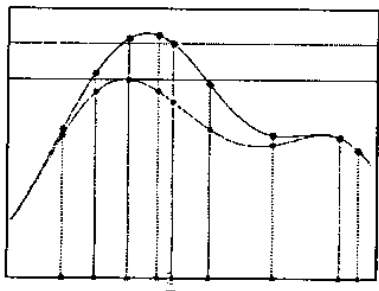

e)

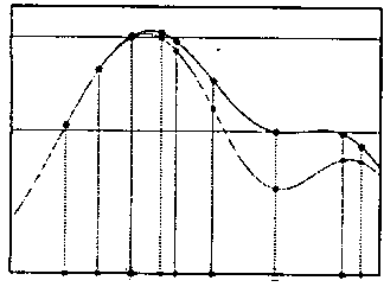

8)

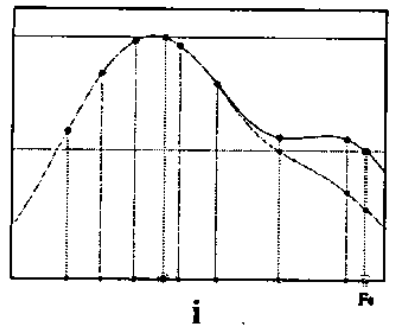

Fig. 3. Illustration of the new filter construction. The supporting window $W$ of size $3 \times 3$ contains 9 pixels of intensities $\{15,24,33,41,45,55,72,90,95\}$.

Each of the graphs from a) to i) shows the dependence of $M_{0}$ and $M_{/ 0}$. $\left(M_{/ 0}<M_{0}\right.$ ), where $M_{/ 0}$ denotes the cumulative similarity value with rejected central pixel $F_{0}$ on the pixel gray scale value. Graph a) shows the plot of $M_{0}$ and $M_{/ 0}$ for $F_{0}=15$, plot b) for $F_{0}=24$ and so on till plot plot i) which shows the graphs of $M_{0}$ and $M_{/ 0}$ for $F_{0}=95$. The central pixel will be replaced in cases: (a), (b), (f)- (i), as in those cases there exists a pixel $F_{i}$ for which $M_{0}<M_{i}$.

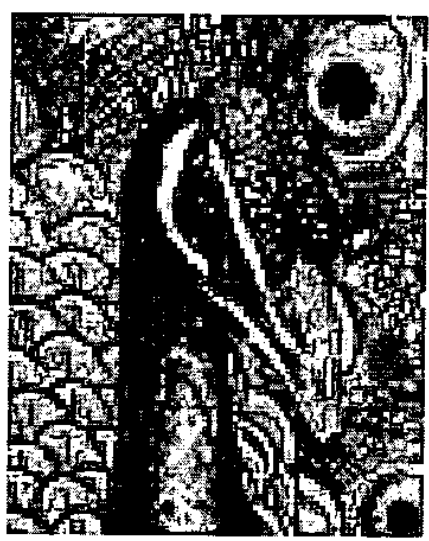

a)

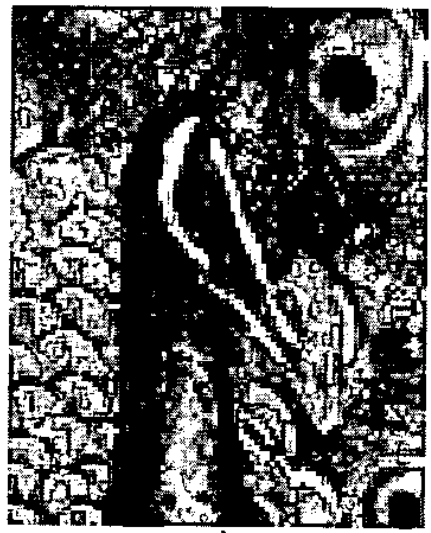

c)

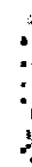

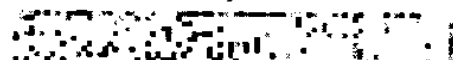

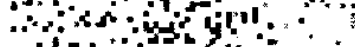

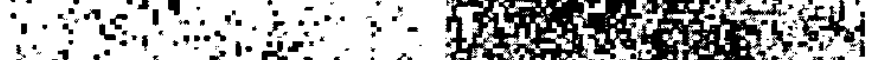
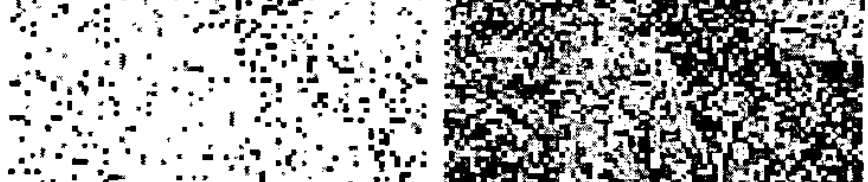

mat

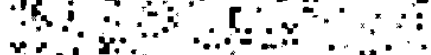

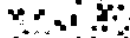

2 .

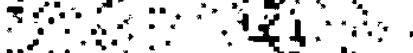

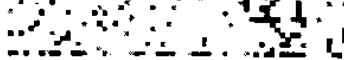

e)

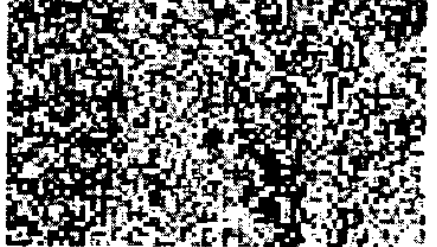

f)

Fig. 4. Comparison of the efficiency of the vector median and the proposed filter: a) test image, b) image contaminated by $4 \%$ impulsive noise, $c$ ) image filtered using the proposed technique, d) the result of the filtering with the vector median, e) and f) depict the filtering error, (absolute value of the difference between the original and filtered image multiplied by 5 ). 